\title{
SEASAFE: an operational tool for sea surface monitoring with SAR
}

\author{
W. Biamino ${ }^{1}$, C. Ananasso ${ }^{2}$, M. Cavagnero ${ }^{1}$, L. Di Matteo ${ }^{1}$, \\ D. Loreggia ${ }^{3}$, F. Tataranni ${ }^{4} \&$ P. Trivero ${ }^{1}$ \\ ${ }^{1}$ Università del Piemonte Orientale "Amedeo Avogadro" - DISIT, Italy \\ ${ }^{2}$ Agenzia Spaziale Italiana, Italy \\ ${ }^{3}$ Istituto Nazionale di Astrofisica, Osservatorio Astrofisico \\ di Torino, Italy \\ ${ }^{4}$ INNOVA Consorzio per l'Informatica e la Telematica, Italy
}

\begin{abstract}
Synthetic Aperture Radar (SAR) is considered as a useful tool for sea surface monitoring, thanks to its capability to acquire images day and night and in all weather conditions. In particular, SAR is extensively used to detect oil slicks on the sea surface. Since the early 90 s SAR acquisitions were routinely available with C-band sensors (such as ERS-1 and 2, Envisat, Radarsat 1 and 2). More recently, also L-band (ALOS PALSAR 1 and 2) and X-band (Terrasar X, COSMO/SkyMed constellation) sensors became operational. In this framework we developed a new software called SEASAFE (Slick Emissions And Ship Automatic Features Extraction), able to process SAR data in C- L- and X-bands. Moving from previous experience, new features have been added such as an enhanced land masking algorithm and oil spill characterization. Modules for ship detection and wind and wave evaluation are also available. The new improved system is here described; detection capabilities are evaluated, overall performances are described.

Keywords: Synthetic Aperture Radar, oil spill, satellite, sea surface monitoring.
\end{abstract}

\section{Introduction}

Oil spills are recognised as a threat to the marine environment. A surface-active material on sea surface increases the damping of short waves. Satellite remote sensing represents a valuable aid for sea surface monitoring and pollution 
prevention. Synthetic Aperture Radar (SAR), which provides radar images day and night and in all weather conditions, is extensively used to monitor the sea surface; in particular, SAR is able to detect sea surface with good accuracy [1]. SAR is able to discriminate surface films under low to moderate wind conditions while high wind conditions are in fact prohibitive. Therefore, it is worthwhile to know the wind field for detection purposes.

Starting from previous experiences [2] we developed a new software named SEASAFE (Slick Emissions And Ship Automatic Features Extraction) with improved features, with the aim to produce a fully operational tool able to analyse C- L-, and X-band satellite SAR images, including COSMO/SkyMed and ALOS PALSAR (1 and 2) to detect and classify sea surface slicks, using (when needed) ancillary data only from publicly accessible repositories, maximizing the amount of information exploitable from SAR data. The software is based on state-of-the art methodologies, found in literature, as well as purposely designed and developed algorithms for some of the features.

The system is intended to be utilised by professional end users for sea observation and pollution prevention purposes; for this reason it does include not only oil spill detection but other features such as oil characterisation, wind evaluation, ship detection. All modules are integrated and intercommunicating. This system is now able to process data in C- L- and X-band from a number of past and present operational satellites.

The main features of SEASAFE are presented in next sections, shortly describing capabilities and issues, with the aim to give an overview about what can be obtained from its use.

\section{Land masking}

Oil spill (OS) detection is generally carried out by considering backscatter values of sea pixels in a SAR image; for this reason inaccuracies in defining coastlines can lead to significant errors in automatic detection of OS near the coast. Also ship detection, in proximity of the coast, can be affected by the same problem.

Commonly, a land mask is obtained by superimposing to the image an archived static coastline; sometimes the shoreline positioning is not accurate because satellite images are often poorly georeferenced without ground control points.

We developed an unsupervised procedure that starts with an archived coastline and change it in order to find better positioning. Among all available archived coastlines we adopted the Global Self-consistent Hierarchical Highresolution Shoreline [3] because of its global coverage, public domain accessibility and adequate resolution.

In our procedure GSHHS is modified to draw actual shorelines on SAR images by evaluating land-sea differences between backscatter values: firstly its positioning is shifted to reach the best overlap and then it is modified by estimating the radiometric intensity gradient in coastal areas of SAR images; small modifications are obtained by applying the Canny edge detection algorithm [4]. The main differences between this approach and other methods 
found in literature are: the simultaneous use of two approaches that improve outcomes, and the coastline search limited to a narrow area across GSHHS, where actual coastline is expected to be, by analysing the backscatter gradient of pixel values.

This approach is well-suited for an unsupervised real-time use, with the aim to replace visual inspection and obtain at least the same results. Coastline detection could be problematic when land and sea show comparable backscattering (e.g. sandy beaches); however, this does not affect the average backscattering calculations, allowing OS detection near the coast.

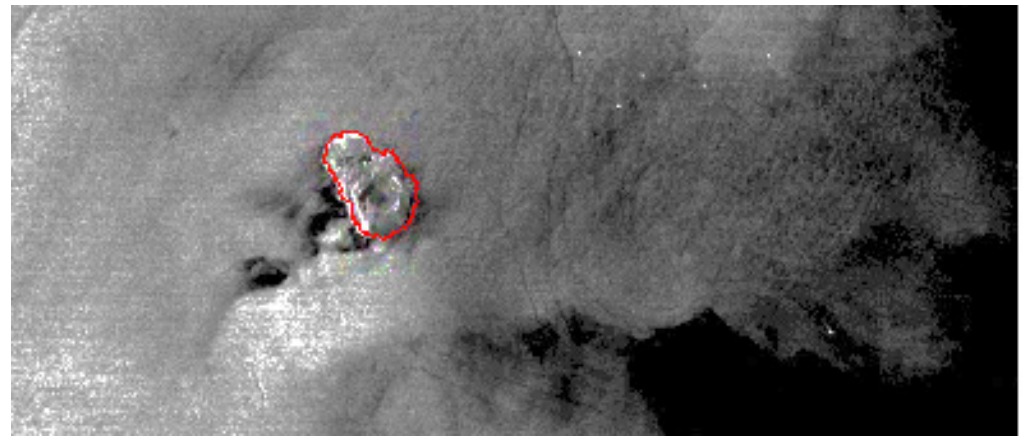

Figure 1: An example of dynamic land masking.

We tested this method on ENVISAT, ERS, RADARSAT, ALOS and COSMO/SkyMed images (see Figure 1) but it is ready to work with other coastlines' databases [5].

\section{Oil slick detection}

The basic approach consists of the following three steps: dark spot detection, dark spot feature extraction and classification of the dark formations as OS or lookalikes (LA). SEASAFE allows to distinguish between OS and LA in supervised or unsupervised way. For every selected candidate we extract, from full resolution image, a rectangular area containing the potential OS with a surrounding sea area, in order to apply a statistical classification procedure.

The unsupervised procedure considers two steps.

Initially, a quicklook image, obtained averaging full resolution image, is scanned to search dark spots that could be "candidate OS". The use of quicklook, instead of processing the whole SAR image, reduces both computation time and memory usage, as well as reduces stronger fluctuations on pixel values.

In the second step, a portion of full resolution image undergoes a classification process to define it as OS or LA. In the supervised procedure an operator can browse the full resolution image and manually define candidates (OS or LA) by selecting small areas on screen.

Examples of OS and LA are shown in Figures 2 and 3. 


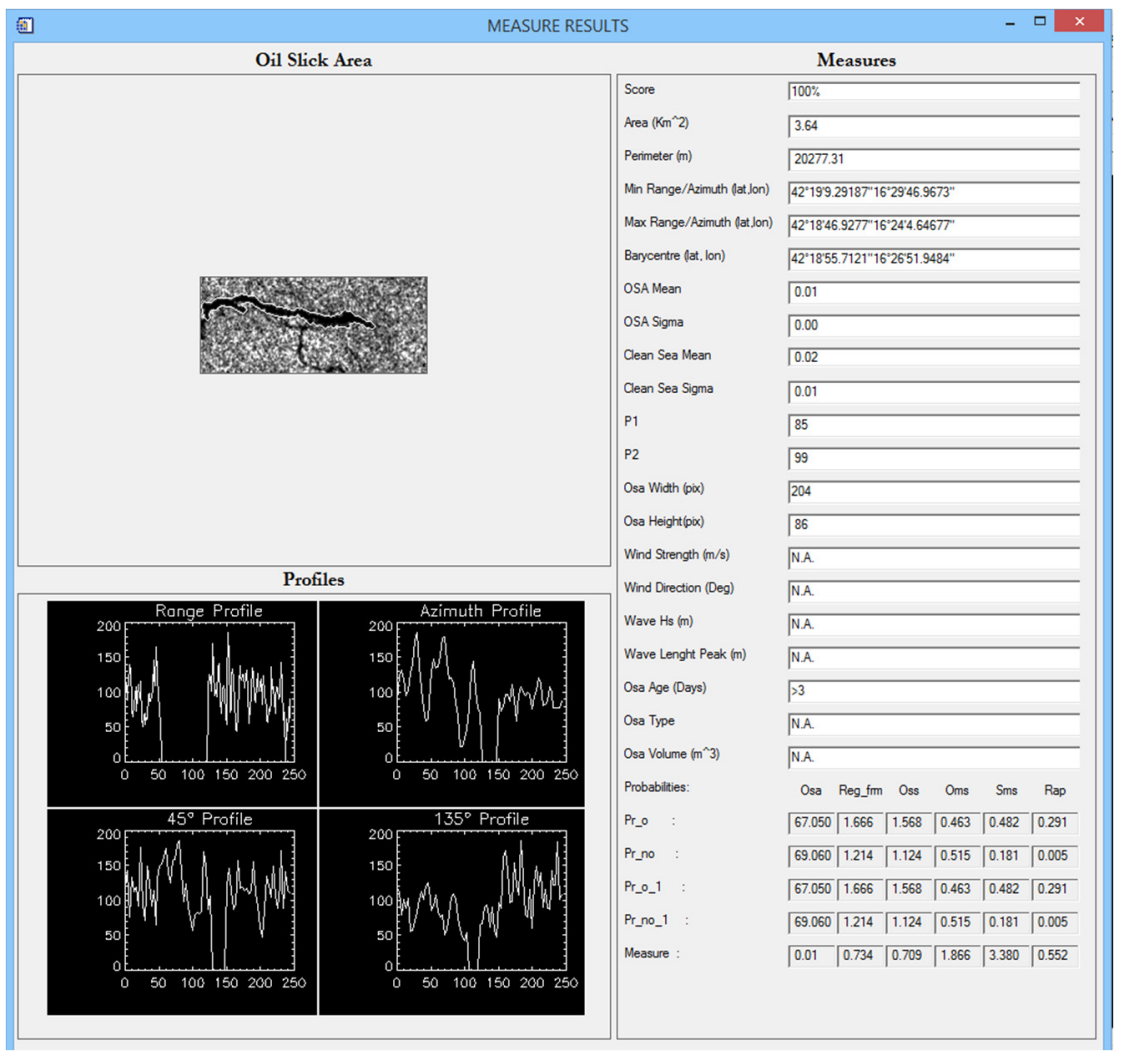

Figure 2: An example of oil spill for COSMO/SkyMed WR image.

In addition to previous detection characteristics, by using the software modules already present in SEASAFE, we have developed a new classification procedure able not only to detect OS, but also to distinguish among different types of LA: conditions of low wind areas, biogenic ("natural") slicks and anthropogenic ("man-made") slicks [6].

One main feature of our approach is that a probability (from $0 \%$ to $100 \%$ ) of being an OS is also provided for each measured dark spot, allowing the operator a better evaluation of the analysed situation.

\section{Wind and wave extraction from SAR images}

The increasing availability of X-band SAR data gathered by the sensors on-board COSMO/SkyMED and TerraSAR-X, encouraged the development of new empirical geophysical models, called XMOD [7-10].

SEASAFE is currently ready to ingest X-band COSMO/SkyMed SAR images for wind extraction purposes; it is also ready to ingest L-band data (PALSAR-1) 


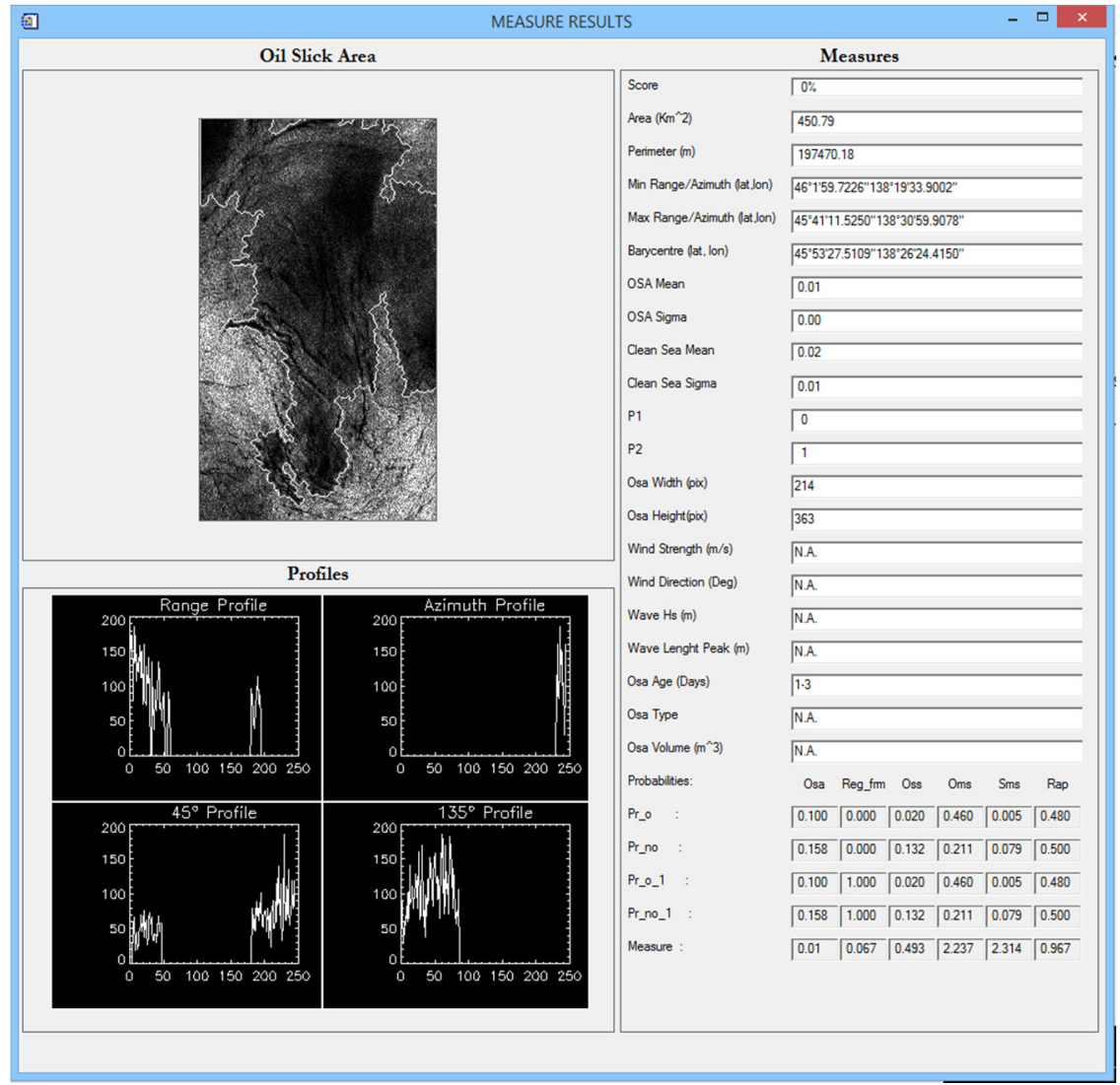

Figure 3: An example of lookalike for Alos WB1 image.

with the algorithm proposed by Isoguchi et al. [11]; tests are ongoing with PALSAR-2 data.

An example of wind field is shown in figure 4.

A new software module to extract bidimensional wave spectrum from SAR image has been developed. This software is useful to evidence the presence of swell waves which modify the overall backscatter and affect the oil spill detectability.

\section{Ship detection}

The ship detection procedure consists of the identification of areas with high brightness (candidate sea object) with respect to the background by a Global Threshold procedure applied on the total image and by an Adaptive Threshold procedure applied to image sub-blocks. For ship detection we adopted a new algorithm based on a CFAR (Constant False Alarm Rate processor): an appropriate PDF (Probability Density Function) is determined which matches the 


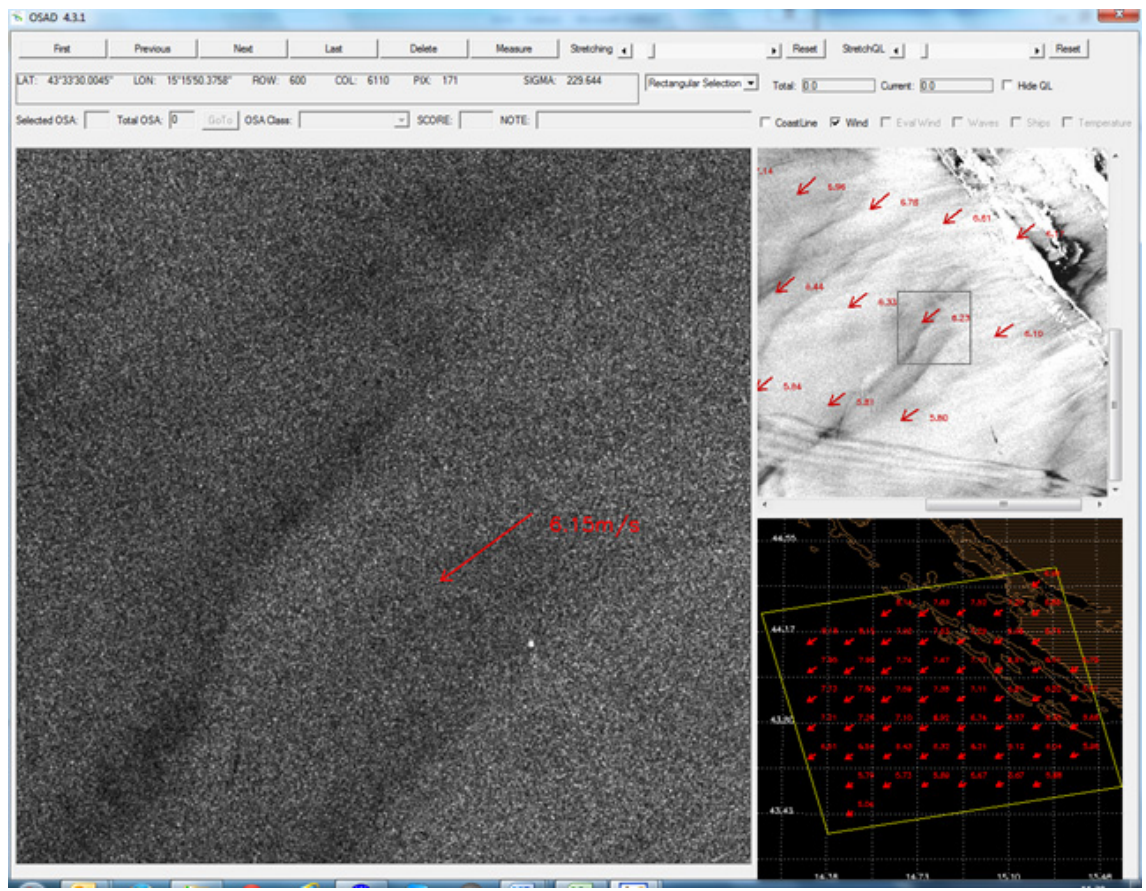

Figure 4: Wind field into SEASAFE.

statistical characteristics of background clutter properly first, then CFAR processor dynamically calculates a detection threshold by the local background distribution and the desired probability of false alarm, and compares the grayscale value of each pixel with the threshold to detect target finally.

The CFAR detector is applied on the image after calibration, in order to equalize the intensity distribution, and land masking; both operations are carried out into SEASAFE.

The resulting calibrated image is then analysed as a whole to localize the areas of interest and then in overlapping blocks selected on the base of the homogeneity and on the resolution of the image.

If necessary, in high noise regime, after a preliminary screening, we apply a correlation filter (that is a correlation kernel for weak signal enhancement) that scroll over all the image in order to find and to amplify weak signals. Then this new image is screened again with a bidimensional cell average method.

The ship detector uses the threshold calculated to discriminate between pixels corresponding to the sea and pixels that potentially contain a floating objects. All the pixels that may be candidate for a ship detection are analysed and, if the size of the candidate area is within a configurable range, that area is retained and flagged as detected target, otherwise the pixels are rejected.

Figure 5 shows a ship correctly identified in a COSMO/SkyMed WR (Look angle at near range $30.13^{\circ}$ look angle at far range $38.76^{\circ}$, pixel spacing $15 \mathrm{~m} \mathrm{x}$ $15 \mathrm{~m}$, spatial resolution $30 \mathrm{~m} \times 30 \mathrm{~m}$ ) image using SEASAFE. In figure 6 a 


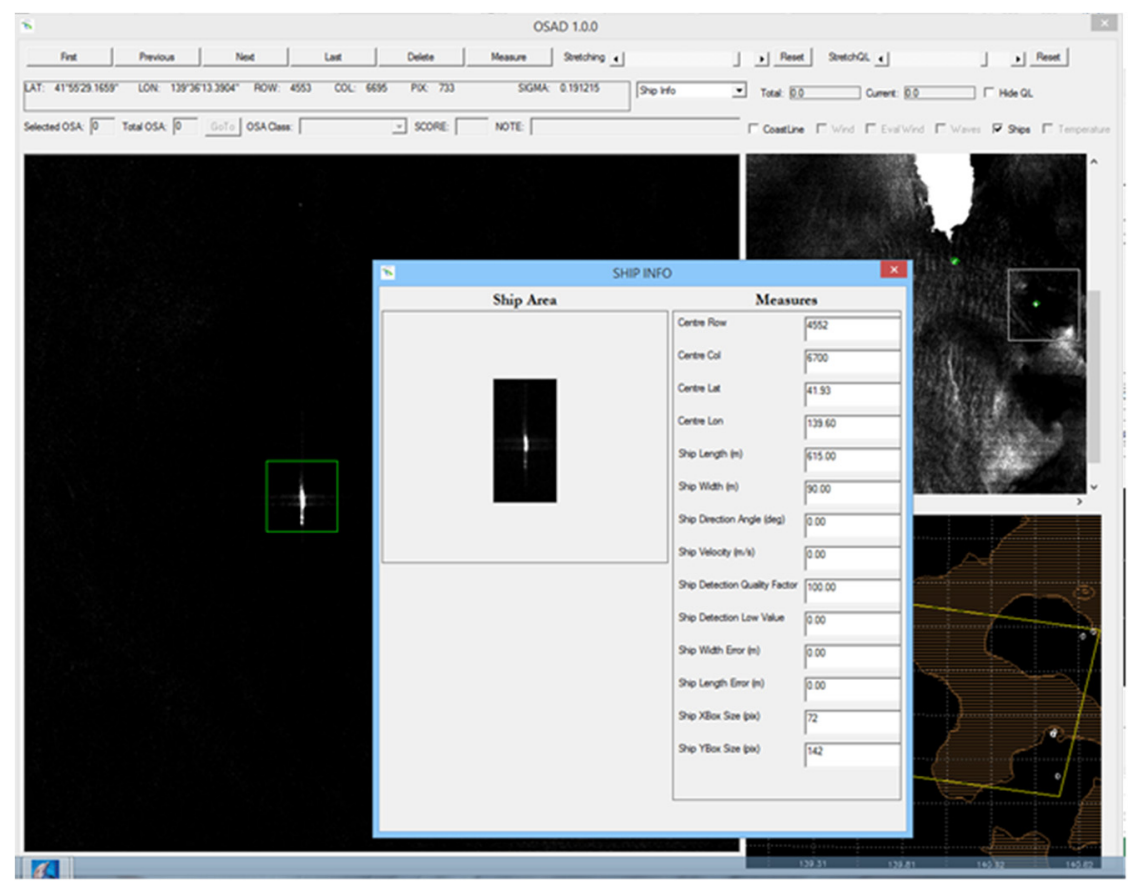

Figure 5: Ship in a COSMO/SkyMed WR image.

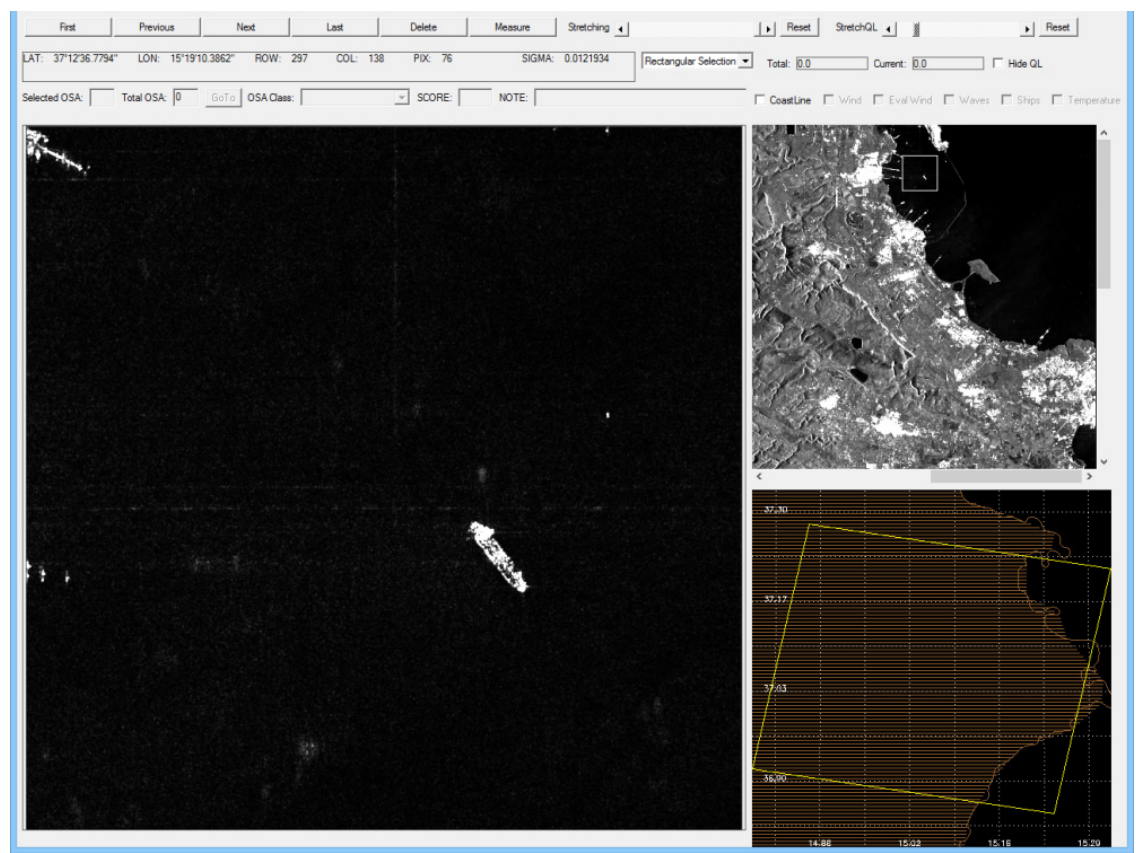

Figure 6: Ship in a COSMO/SkyMed HI image (analysed with SEASAFE). 
COSMO/SkyMed HI image is shown to evidence how a big ship can be clearly seen by SAR.

With regards to other existing ship detection approaches, this method is specially focused to the detection of small objects.

\section{Oil spill characterization}

During emergency episodes, the knowledge of oil type and spill age is usually provided by aerial visual inspection, if and when available. We propose a prototypal characterisation algorithm based on SAR data.

Geometric and radiometric intensity from both the OS and the surrounding clean sea are used in our procedure. Moreover, we also take into account the OS weathering due to meteorological parameters (wind and wave fields, extracted from the SAR image itself).

The rationale of characterisation algorithm is based on information from literature and experimental evidence. First, we assume that age, thickness, and oil composition are not completely independent. For example, an older slick usually is thinner (owing to oil diffusion); this process is slower for heavier oils, and therefore a slick of this type will be thicker at the same age. Wind action is included, and its effect is more noticeable as the OS ages. Wind effects on OS are jagging, thickness gradients, and reduced overall thickness.

On an observational basis, detected OS were evaluated to categorise every OS by age class:

- Recent ( $<1$ day): linear contour, uniform black colour;

- Medium (1-3 days): "intermediate" features (between the other two classes);

- Old (>3 days): jagged contour, noticeable grey shades in slick appearance.

OS composition is assessed on the basis of observation: heavier oils usually show well-defined and smooth borders; radiometric intensity measured along the wind direction shows a uniform damping; the intensity gradient on the border is similar on both sides. A lighter OS easily breaks up.

OS thickness is estimated by combining age and composition data. Finally, the OS volume is evaluated. The outputs are classified is three classes:

a) up to $10 \mathrm{~m}^{3}$;

b) 10 to $100 \mathrm{~m}^{3}$;

c) more than $100 \mathrm{~m}^{3}$.

The algorithm was calibrated with measurements campaign.

Figure 7 shows a sample of SEASAFE output on an OS with the evaluated parameters: age, type, and volume (mass); here the spill covers an area of $7 \mathrm{~km} \times$ $7 \mathrm{~km}^{2}$. 

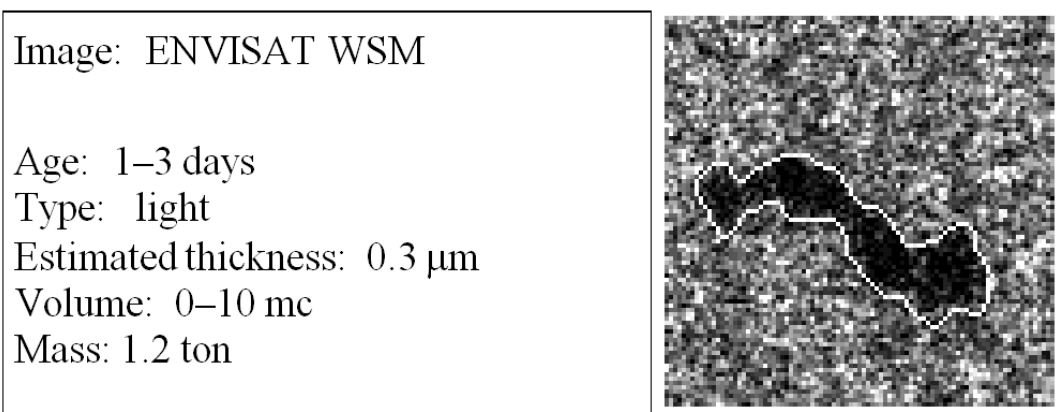

Figure 7: Example of SEASAFE oil characterization.

\section{Conclusions}

The operational system SEASAFE we developed can automatically detect OS from all types of SAR images (all operating modes, all polarisations) and distinguish between real OS and LA. The probability of being an OS is also provided.

The SAR capability of detecting OS has produced favourable results in the open sea, even using low resolution. In sheltered areas, system sensitivity was improved using the training dataset and an effective land masking was added to build an OS survey system suitable for application to small basins or near coasts.

Wind and wave fields are estimated from $\mathrm{C}$ - and X-band SAR images, while extension to L-band is ongoing. Wind is then used in a first prototypal characterisation algorithm; wind and wave data are useful for oil drift simulation as well as for intervention planning.

A ship detection module is available, specialised for small floating objects, with the aim to link a detected slick to the possible source. Moreover, areas with ships (that appear brighter in SAR image) are temporarily excluded from the computations for oil slick detection, in order to improve calculations.

The system tuning can be continuously improved by adding measurements, from certified OS, to statistical analyses. The software is ready to use in case of emergencies, with satellite images in standard format.

\section{Acknowledgements}

This work was developed as part of the ASI project PRIMI (Grant no. I/094/06/0) and was supported by ASI. Further development is ongoing in the framework of the "ASI-JAXA: SAR data exploiting for oil pollution detection in coastal areas" project (Grant no. 2013-037-R.0). This work has been partially supported by the RITMARE Flagship Project funded by Italian Ministry of University and Research. This work has been developed during the ASI project PRIMI (contract n. I/094/06/0) and was supported by ASI. ALOS-1 data (CJAXA - all rights reserved) and COSMO/SkyMed data (CASI - all rights reserved) have been obtained in the framework of the two mentioned projects. 


\section{References}

[1] Trivero, P., Fiscella, B. \& Pavese, P., Sea surface slicks measured by SAR. Il Nuovo Cimento della Società Italiana di Fisica. C. Geophysics and space physics, 24(1), pp. 99-111, 2001.

[2] Nirchio, F., Sorgente, M., Giancaspro, A., Biamino, W., Parisato, E., Ravera, R. \& Trivero, P., Automatic detection of oil spills from SAR images, International Journal of Remote Sensing, 26(6), pp. 1157-1174, 2005.

[3] Wessel, P. \& Smith, W.H.F., A global, self-consistent, hierarchical, high-resolution shoreline database. Journal of Geophysical Research, 101, pp. 8741-8743, 1996.

[4] Canny, J., A computational approach to edge detection, IEEE Transactions on Pattern Analysis and Machine Intelligence, 8(6), pp. 679698, 1986.

[5] Biamino, W., Borasi, M., Cavagnero, M., Croce, A., Di Matteo, L., Fontebasso, F., Tataranni, F. \& Trivero, P., A "dynamic" land masking algorithm for synthetic aperture radar images, Proc. of IGARSS 2015 conference, Institute of Electrical and Electronics Engineers: New York, in press.

[6] Trivero, P., Biamino, W., Borasi, M., Cavagnero M., Di Matteo L. \& Loreggia, D., Sea slicks classification by Synthetic Aperture Radar, Proc. of SPIE remote sensing 2014 conference, International Society for Optics and Photonics: Bellingham, pp. 92400C-92400C, 2014.

[7] Nirchio, F. \& Venafra, S., Preliminary model for wind estimation from Cosmo/SkyMed X band SAR data, Proc. of IGARSS 2010 conference, Institute of Electrical and Electronics Engineers: New York, pp. 3462 3465, 2010.

[8] Ren, Y, Lehner, S., Brusch, S., Li, X. \& He, M., An algorithm for the retrieval of sea surface wind fields using X-band TerraSAR-X data, International Journal of Remote Sensing, 33(23), pp. 7310-7336, 2012.

[9] Nirchio, F. \& Venafra, S., XMOD2 - An improved geophysical model function to retrieve sea surface wind fields from Cosmo SkyMed X-band data, European Journal of Remote Sensing, 46, pp. 583-595, 2013.

[10] Li, X. \&, Lehner S., Algorithm for sea surface wind retrieval from TerraSAR-X and TanDEM-X Data, IEEE Transactions on Geoscience and Remote Sensing, 52(5), pp. 2928-2939, 2014.

[11] Isoguchi, O. \& Shimada, M., An L-Band ocean geophysical model function derived from PALSAR, IEEE Transactions on Geoscience and Remote Sensing, 47(7), 1925-1936, 2009. 